\title{
Synthesis and antimicrobial activity of Mannich bases of isatin and its derivatives with 2-[(2,6-dichlorophenyl)amino]phenylacetic acid
}

\author{
V. Ravichandran, ${ }^{\dagger}$ S. Mohan, and K. Suresh Kumar
}

K. M. C. H. College of Pharmacy, Coimbatore, Tamilnadu, India 641035

E-mail:Phravi75@,rediffmail.com

\begin{abstract}
A series of 2,3-dihydro-2-oxo-1,3-disubstituted indoles were prepared by the reaction of 2,3dihydro-2-oxo-3-substituted indoles with 2-[(2,6-dichlorophenyl)amino]phenylacetic acid in the presence of formaldehyde. The newly synthesized compounds were characterized on the basis of elemental analysis, IR, ${ }^{1} \mathrm{H}$ NMR and mass spectra. All the synthesized compounds were tested for their antibacterial activities against Gram + and Gram - bacteria, and antifungal activities. Among the synthesized compounds RS II showed moderate antibacterial activity against $B$. subtilis, RS I, RS II, RS III and RS VI showed moderate antibacterial activity against $P$. aeruginosa, RS V and RS VI showed good anti fungal activity against $P$. notatum.
\end{abstract}

Keywords: Isatin, Schiff base, Mannich base, antimicrobial activity

\section{Introduction}

Isatin (2,3-dioxindole) is an endogenous compound with a long history and wide range of pharmacological actions. $\mathrm{N}$-alkyl isatin derivatives were synthesized and screened against Gram + and Gram - bacteria [1]. Schiff and Mannich bases of isatin and its derivatives were synthesized and reported for antibacterial [2], antifungal [3], anti-HIV [4-11], anticonvulsant activities [12] and GAL3 receptor antagonists [13,14]. Prompted by the biological properties of 2,3-dioxindole derivatives and its Schiff and Mannich bases, it was decided to synthesize various Mannich bases of isatin and its derivatives with 2-[(2,6-dichlorophenyl) amino] phenyl acetic acid and to screen them for their antimicrobial properties. The results of such studies are discussed in this paper.

\footnotetext{
${ }^{\dagger}$ Present address: Department of Pharmaceutical Sciences, Dr. H. S. Gour Vishwavidyalaya, Sagar (M.P), India 470003
} 


\section{Results and Discussion}

In the present study, Schiff bases of isatin were synthesized by condensation of the keto group of isatin with different aromatic primary amines. The N-Mannich bases of the above Schiff bases were synthesized by reaction of the acidic imino group of isatin with formaldehyde and 2-[(2,6dichlorophenyl)amino]phenylacetic acid. The previous reports [2-10] revealed that Mannich bases of isatin with substituted aromatic amines show good antimicrobial activity, especially halogenated aromatic amines; therefore, we have selected 2-[(2,6dichlorophenyl)amino]phenylacetic acid to make Mannich bases with isatin in the present study. All the compounds gave satisfactory elemental analysis (Table 1). IR, ${ }^{1} \mathrm{H}$ NMR and mass spectra were consistent with the assigned structures.

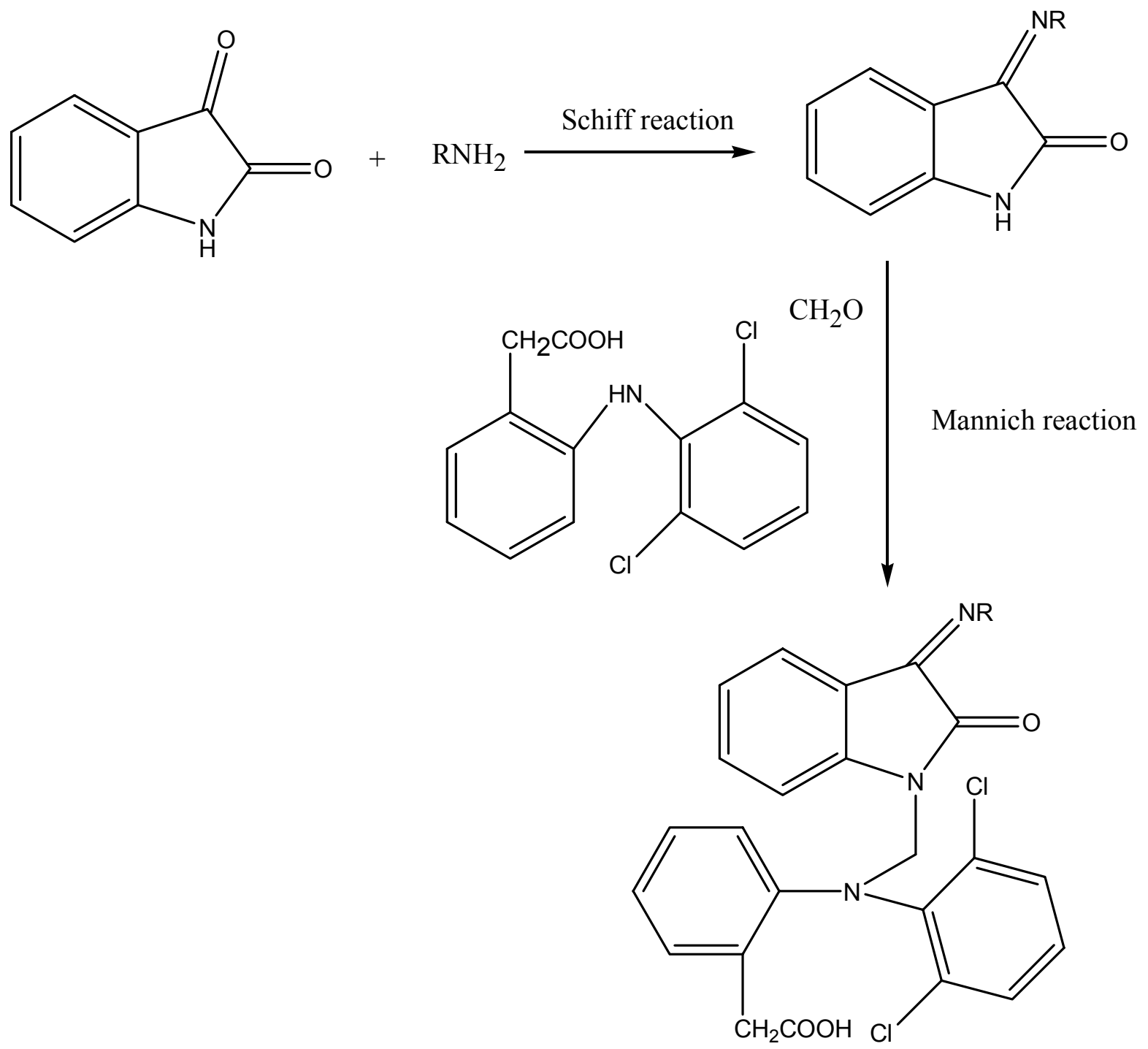

Scheme 1. Synthesis of the studied compounds. 
Table 1. The physical constant of the synthesized compounds

\begin{tabular}{|c|c|c|c|c|c|}
\hline \multirow[t]{2}{*}{ Compd } & \multirow{2}{*}{$\mathrm{R}$} & \multirow{2}{*}{$\begin{array}{l}\text { Molecular } \\
\text { formula }\end{array}$} & \multicolumn{3}{|c|}{$\begin{array}{l}\text { Elemental analysis } \\
\text { Found/calculated (\%) }\end{array}$} \\
\hline & & & C & $\mathrm{H}$ & $\mathrm{N}$ \\
\hline RS I & & $\mathrm{C}_{29} \mathrm{H}_{20} \mathrm{Cl}_{3} \mathrm{~N}_{3} \mathrm{O}_{3}$ & $\begin{array}{l}61.35 \\
61.66\end{array}$ & $\begin{array}{l}3.46 \\
3.57\end{array}$ & $\begin{array}{l}7.35 \\
7.44\end{array}$ \\
\hline RS II & & $\mathrm{C}_{29} \mathrm{H}_{22} \mathrm{Cl}_{2} \mathrm{~N}_{4} \mathrm{SO}_{5}$ & $\begin{array}{l}57.16 \\
57.15\end{array}$ & $\begin{array}{l}3.54 \\
3.64\end{array}$ & $\begin{array}{l}9.17 \\
9.19\end{array}$ \\
\hline RS III & & $\mathrm{C}_{30} \mathrm{H}_{21} \mathrm{Cl}_{2} \mathrm{~N}_{3} \mathrm{O}_{5}$ & $\begin{array}{l}62.70 \\
62.73\end{array}$ & $\begin{array}{c}3.65 \\
3.68\end{array}$ & $\begin{array}{l}7.30 \\
7.32\end{array}$ \\
\hline RS IV & & $\mathrm{C}_{30} \mathrm{H}_{21} \mathrm{Cl}_{2} \mathrm{~N}_{3} \mathrm{O}_{5}$ & $\begin{array}{l}62.68 \\
62.73\end{array}$ & $\begin{array}{l}3.65 \\
3.68\end{array}$ & $\begin{array}{l}7.32 \\
7.32\end{array}$ \\
\hline RS V & & $\mathrm{C}_{29} \mathrm{H}_{22} \mathrm{Cl}_{2} \mathrm{~N}_{4} \mathrm{O}_{3}$ & $\begin{array}{l}63.84 \\
63.86\end{array}$ & $\begin{array}{l}4.02 \\
4.07\end{array}$ & $\begin{array}{l}10.28 \\
10.27\end{array}$ \\
\hline RS VI & & $\mathrm{C}_{23} \mathrm{H}_{16} \mathrm{Cl}_{2} \mathrm{~N}_{2} \mathrm{O}_{4}$ & $\begin{array}{l}60.63 \\
60.67\end{array}$ & $\begin{array}{l}3.52 \\
3.54\end{array}$ & $\begin{array}{l}6.11 \\
6.15 \\
\end{array}$ \\
\hline
\end{tabular}

All the synthesized compounds were tested for in vitro antimicrobial activity. The MIC values of the compounds against pathogenic bacteria and fungi are presented in Table 2. All compounds have shown moderate activity, but not up to our expectation, against tested bacteria and fungi. It may be, because of the bulky phenyl acetic acid group present at nitrogen atom of isatin nucleus. This bulky group may sterically hinder the compounds binding with bacteria. Substitution of isatin at 3-position with a substituted phenyl ring is conducive for antibacterial activity compared to an unsubstituted phenyl group or no substitution at the same position of isatin. Moreover, compound RS II showed good activity against all the bacteria tested suggesting that the substitution of isatin at the 3-position with a substituted phenyl ring favors antibacterial activity compared to an unsubstituted phenyl ring at 
the same position. The antifungal activity of the compounds was studied with pathogenic fungi, and the results are given in Table 2. Fluconazole is used as a reference. All the compounds showed significant antifungal activity, especially compound RS V, indicating that unsubstituted phenyl ring at 3-position of isatin is desired for anti-fungal activity compared to substituted phenyl ring.

Table 2. Antimicrobial activity of the compounds MIC's in $\mu \mathrm{g} / \mathrm{mL}^{\mathrm{a}}$

\begin{tabular}{|c|c|c|c|c|c|c|c|c|}
\hline \multirow[b]{2}{*}{ Microorganisms } & \multicolumn{6}{|c|}{ Compounds } & \multirow[b]{2}{*}{ Ciprofloxacin } & \multirow[b]{2}{*}{$\begin{array}{c}\text { Fluconazol } \\
\mathrm{e}\end{array}$} \\
\hline & RS I & RS II & RS III & RS IV & RS V & RS VI & & \\
\hline \multicolumn{9}{|c|}{ MIC Value } \\
\hline Escherchia coli & 25.0 & 25.0 & 50.0 & 25.0 & 50.0 & 25.0 & 3.9 & -- \\
\hline $\begin{array}{c}\text { Pseudomonas } \\
\text { aeruginosa }\end{array}$ & 12.5 & 12.5 & 12.5 & 50.0 & 25.0 & 25.0 & 3.9 & -- \\
\hline Bacillus cereus & 25.0 & 25.0 & 50.0 & 50.0 & 50.0 & 50.0 & 7.8 & -- \\
\hline Bacillus subtilis & 25.0 & 12.5 & 50.0 & 50.0 & 25.0 & 50.0 & 3.9 & -- \\
\hline $\begin{array}{l}\text { Klebsiella } \\
\text { aeruginosa }\end{array}$ & 50.0 & 50.0 & 50.0 & 50.0 & 25.0 & 50.0 & 15.6 & -- \\
\hline $\begin{array}{c}\text { Staphylococcus } \\
\text { aureus }\end{array}$ & 12.5 & 25.0 & 25.0 & 50.0 & 25.0 & 50.0 & 3.9 & -- \\
\hline $\begin{array}{c}\text { Penicillium } \\
\text { notatum }\end{array}$ & 50.0 & 50.0 & 100.0 & 12.5 & 6.25 & 12.25 & -- & 2.5 \\
\hline Candida albicans & 100.0 & 50.0 & 100.0 & 100.0 & 100.0 & 100.0 & -- & 10.0 \\
\hline Aspergillus niger & 12.5 & 25.0 & 50.0 & 50.0 & 12.5 & 25.5 & -- & 5.0 \\
\hline
\end{tabular}

${ }^{a}$ MIC- Minimum inhibitory concentration.

\section{Experimental Section}

\section{Chemistry}

General Procedures. Melting points were determined using an open-ended capillary method and are uncorrected. The purity of the synthesized compounds was checked by TLC. UV/VIS spectra were taken in a Schimadzu UV/VIS 1700 spectrophotometer. FT-IR was recorded on a Jasco FT-IR spectrophotometer, ${ }^{1} \mathrm{H}$ NMR spectra were recorded at $300 \mathrm{MHZ}$ on a Bruker FTNMR spectrophotometer and mass spectra on a Varian Atlas $\mathrm{CH}-7$ mass spectrometer at $70 \mathrm{ev}$ (IICT, Hydrabad). The elemental analysis was obtained on a Vario-EL instrument. 


\section{General synthesis of 2,3-dihydro-2-oxo-1,3-disubstituted indoles}

Isatin was allowed to react with different aromatic primary amines like 4-chloroaniline, sulfanilamide, 4-amino benzoic acid, 2-amino benzoic acid and phenyl hydrazine respectively, in the presence of absolute alcohol and the $\mathrm{pH}$ was adjusted to 4-5 with glacial acetic acid to get 2,3-dihydro-2-oxo-3-substituted indoles. These compounds were directly used for the next step.

The Mannich reactions of 2,3-dihydro-2-oxo-3-substituted indoles with 2-[(2,6dihydrophenyl)amino]phenylacetic acid in the presence of formaldehyde were carried out at 0-5 ${ }^{\circ} \mathrm{C}$ by stirring the reaction mixture with magnetic stirrer. The reactions yielded 2,3-dihydro-2oxo-1,3-disubstituted indoles (Scheme 1). The synthesized compounds were recrystalized from hot ethanol. The physical characteristics of the synthesized compounds are listed in Table 1.

\section{Spectral data}

\{2-[3-(4-Chlorophenylimino)-2-oxo-2,3-dihydro-indol-1-yl-methyl]-(2,6-dichlorophenyl) amino]phenyl acetic acid. (RS I). Yield 43.5\%, mp $177-179{ }^{\circ} \mathrm{C}$, UV $\left(\lambda_{\max }\right) 420.5 \mathrm{~nm}$, IR $(\mathrm{KBr}) \mathrm{V}_{\max }$ in $\mathrm{cm}^{-1} 3414(\mathrm{O}-\mathrm{H}), 1616(\mathrm{C}=\mathrm{N}), 1509$ (characteristic of 1,2-disubstituted benzene), $749(\mathrm{C}-\mathrm{Cl}) .{ }^{1} \mathrm{H}-\mathrm{NMR}$ (acetone) $\delta \mathrm{ppm}: 3.52\left(\mathrm{~s}, 2 \mathrm{H},-\mathrm{CH}_{2}-\mathrm{COO}\right), 4.75(\mathrm{~s}, 2 \mathrm{H}, \mathrm{N}-$ $\left.\mathrm{CH}_{2}-\mathrm{N}\right), 6.40-6.91(\mathrm{~m}, 7 \mathrm{H}, \mathrm{Ar}-\mathrm{H}), 7.21-7.63(\mathrm{~m}, 8 \mathrm{H}, \mathrm{Ar}-\mathrm{H}) .11 .0$ (s, 1H, -COOH). EI-MS $(\mathrm{m} / \mathrm{z}) 565$.

(2-\{(2,6-Dichlorophenyl)-[2-oxo-3-(4-sulfamoylphenylimino)-2,3-dihydroindol-1-yl-methyl] amino\}phenyl)acetic acid. (RS II). Yield 76\%, mp 260-262 ${ }^{\circ} \mathrm{C}, \mathrm{UV}\left(\lambda_{\max }\right) 414.5 \mathrm{~nm}, \mathrm{IR}(\mathrm{KBr})$ $\mathrm{v}_{\max }$ in $\mathrm{cm}^{-1} 3411(\mathrm{O}-\mathrm{H}), 1737(\mathrm{C}=\mathrm{O}), 1617(\mathrm{C}=\mathrm{N}), 1150\left(\mathrm{SO}_{2}\right), 749(\mathrm{C}-\mathrm{Cl}) .{ }^{1} \mathrm{H}-\mathrm{NMR}$ (acetone) $\delta$ ppm: 2.0 (br, 2H, $\mathrm{SO}_{2} \mathrm{NH}_{2}$ ), 3.52 (s, 2H, $\left.-\mathrm{CH}_{2}-\mathrm{COO}\right), 4.72$ (s, 2H, N-CH$-\mathrm{N}$ ), 6.43-6.90 (m, 7H, Ar-H), 7.20-7.65 (m, 8H, Ar-H). 11.20 (s, 1H, -COOH).

4-(1-\{[(2-Carboxymethylphenyl)-(2,6-dichlorophenyl)amino]methyl\}-2-oxo-1,2-dihydroindol-3-ylidine amino)benzoic acid. (RS III). Yield 47.4\%, mp 150-152 ${ }^{\circ} \mathrm{C}$, UV $\left(\lambda_{\max }\right) 363.5$ $\mathrm{nm}, \mathrm{IR}(\mathrm{KBr}) \mathrm{v}_{\max }$ in $\mathrm{cm}^{-1} 3413(\mathrm{O}-\mathrm{H}), 1737(\mathrm{C}=\mathrm{O}), 1504$ (characteristic of 1,2-disubstituted benzene). ${ }^{1} \mathrm{H}-\mathrm{NMR}$ (acetone) $\delta \mathrm{ppm}: 3.61$ (s, 2H, - $\left.\mathrm{CH}_{2}-\mathrm{COO}\right), 4.65$ (s, 2H, N-CH2-N), 6.51-6.86 (m, 7H, Ar-H), 7.12-7.60 (m, 8H, Ar-H), 11.42 (s, 1H, -COOH). EI-MS (m/z) 574.

2-(1-\{(2-Carboxymethylphenyl)-(2,6-dichlorophenyl)amino)methyl\}-2-oxo-1,2-dihydroindol-3-ylidine amino)benzoic acid. (RS IV). Yield 77.8\%, mp 155-157 ${ }^{\circ} \mathrm{C}$, UV $\left(\lambda_{\max }\right) 291.4 \mathrm{~nm}$, IR $(\mathrm{KBr}) \mathrm{v}_{\text {max }}$ in $\mathrm{cm}^{-1} 3415(\mathrm{O}-\mathrm{H}), 1616(\mathrm{C}=\mathrm{N}), 1508$ (characteristic of 1,2-disubstituted benzene). ${ }^{1} \mathrm{H}-\mathrm{NMR}$ (acetone) $\delta$ ppm: 3.60 (s, 2H, - $\mathrm{CH}_{2}-\mathrm{COO}$ ), 4.62 (s, 2H, N-CH $-\mathrm{N}$ ), 6.50-6.81 (m, 7H, ArH), 7.21-7.65 (m, 8H, Ar-H), 11.0 (s, 1H, COOH). EI-MS (m/z) 574.

(2-\{(2,6-Dihydrophenyl)-[2-oxo-3-(phenylhydrazono)-2,3-dihydroindol-1-ylmethyl]amino\} phenyl)acetic acid. (RS V). Yield 55.5\%, mp 160-162 ${ }^{\circ} \mathrm{C}, \mathrm{UV}\left(\lambda_{\max }\right) 435 \mathrm{~nm}, \mathrm{IR}(\mathrm{KBr}) \mathrm{V}_{\max }$ in $\mathrm{cm}^{-1} 3413(\mathrm{O}-\mathrm{H}), 1575(\mathrm{C}=\mathrm{N}), 1507$ (characteristic of 1,2-disubstituted benzene), $1391(\mathrm{C}=\mathrm{O}) .{ }^{1} \mathrm{H}-$ NMR (Acetone) $\delta$ ppm: 3.63 (s, 2H, $\left.-\mathrm{CH}_{2}-\mathrm{COO}\right), 4.60$ (s, 2H, N-CH$\left.-\mathrm{N}\right), 6.23-6.81$ (m, 7H, Ar-H), 7.0 (s, 1H, -NH-), 7.30-7.86 (m, 9H, Ar-H), 11.21 (s, 1H, -COOH). EI-MS (m/z) 544. 


\section{\{2-[(2,6-Dichlorophenyl)-(2,3-dioxo-2,3-dihydroindol-1-yl-methyl)amino]phenyl\}acetic acid. (RS} VI).

Yield 62.3\%, mp 158-160 ${ }^{\circ} \mathrm{C}$, UV $\left(\lambda_{\max }\right) 413.5 \mathrm{~nm}$, IR $(\mathrm{KBr}) \mathrm{v}_{\max }$ in $\mathrm{cm}^{-1} 3413(\mathrm{O}-\mathrm{H}), 1738$ $(\mathrm{C}=\mathrm{O}), 1616(\mathrm{C}=\mathrm{N}) .{ }^{1} \mathrm{H}-\mathrm{NMR}$ (acetone) $\delta \mathrm{ppm}: 3.60\left(\mathrm{~s}, 2 \mathrm{H},-\mathrm{CH}_{2}-\mathrm{COO}\right), 4.62\left(\mathrm{~s}, 2 \mathrm{H}, \mathrm{N}-\mathrm{CH}_{2}-\mathrm{N}\right)$, 6.80-7.64 (m, 11H, Ar-H), 10.92 (s, 1H, -COOH).

\section{Biological evaluation}

\section{In vitro antibacterial activity}

Synthesized compounds were evaluated for their in-vitro antibacterial activity against pathogenic bacteria. The agar dilution method was performed using Muller-Hinton agar (Hi-Media) medium. Suspension of each microorganism was prepared and applied to plates with serially diluted compounds (DMF, solvent control) to be tested and incubated (approx. $20 \mathrm{~h}$ ) at $37{ }^{\circ} \mathrm{C}$. The minimum inhibitory concentration (MIC) was considered to be the lowest concentration that was completely inhibited growth on agar plates. The bacteria strains Pseudomonas aeruginosa (NCIM 2200), Staphylococcus aureus (NCIM 2079), Escherichia coli (NCIM 2065), Bacillus subtilis (NCIM 2063), Bacillus cereus (NCIM 2155) and Klebsiella aeruginosa (NCIM 2239) were used for this study.

\section{In vitro antifungal activity}

The compounds were evaluated for their in vitro anti-fungal activity against pathogenic fungi using agar dilution method with Saburoud's dextrose agar (Hi-Media). Suspension of each fungus were prepared and applied to agar plates with serially diluted compounds to be tested. The plates were incubated at $26^{\circ} \mathrm{C}$ for $72 \mathrm{~h}$ and MIC's were determined. The fungus strains $C$. albicans (NCIM 3102) and P. notatum (NCIM 742) were used for this study.

\section{Acknowledgements}

The authors are thankful to Prof. Abhay Dharamsi, Principal, KMCH College of Pharmacy, Coimbatore for providing the necessary facilities to carry out this work.

\section{References}

1. Pandeya, S. N.; Sriram, D.; Nath, G.; DeClercq, E. Farmaco, 1999, 54, 624.

2. Daisley, R. W.; Shah, V. K. J. Pharm. Sci., 1984, 73, 407.

3. Piscopo, B.; Diumo, M. V.; Godliardi, R.; Cucciniello, M.; Veneruso, G. Bol. Soc. Ital. Biol. Sper., 1987, 63, 827.

4. Teitz, Y.; Ronen, D.; Vansover, A.; Stematsky, T.; Rigg, J. L. Antiviral Res., 1994, 24, 215.

5. Pandeya, S. N.; Sriram, D.; DeClercq, E.; Pannecouque, C.; Witvrouw, M. Indian J. Pharm. Sci., 1998, 60, 207. 
6. Pandeya, S. N.; Sriram, D.; DeClercq, E.; Nath, G. Eur. J. Pharm. Sci., 1999, 9, 25.

7. Pandeya, S. N.; Sriram, D.; DeClercq, E.; Nath, G. Pharm. Acta. Helv., 1999, 74, 11.

8. Pandeya, S. N.; Yogeswari, P.; Sriram, D.; DeClercq, E.; Pannecouque, C.; Witvrouw, M. Chemotherapy, 1999, 45, 192.

9. Pandeya, S. N.; Sriram, D.; DeClercq, E.; Nath, G. Arzneim Forsch., 2000, 50, 55.

10. Selvam, P.; Chandramohan, M.; DeClercq, E.; Witvrouw, M.; Pannecouque, C. Eur. J.

11. Pharm. Sci., 2001, 14, 313.

12. Gursoy, A.; Karali, N.; Buyuktimakin, S.; Demirayak, S.; Ekinci, A. C.; Ozer, H. Farmaco, 1996, 39, 5072.

13. Konkel, M. J.; Lagu, B.; Boteju, L. W.; Jimenez, H.; Noble, S.; Walker, M. W.; Chandrasena, G.; Blackburn, T. P.; Nikam, S. S.; Wright, J. L.; Kornberg, B. E.; Gregory, T.; Pugsley, T. A.; Akunne, H.; Zoski, K.; Wise, L. D. J. Med. Chem., 2006, 49, 3757.

14. Konkel, M. J.; Packiarajan, M.; Chen, H.; Topiwala, U. P.; Jimenez, H.; Talisman, I. J.; Coate, H.; Walker, M. W. Bioorg. Med. Chem. Lett., 2006, 16, 3950. 